\title{
Light Source Position Microsensor
}

\author{
J.M. Quero, A. Guerrero and L.G. \\ Franquelo \\ Dpto. Ingeniería Electrónica \\ E.S. Ingenieros de Sevilla \\ e-mail: quero@esi.us.es
}

\begin{abstract}
There exist industrial applications where an accurate estimation of a light source position is needed. That is the case of a heliostat, a device that projects sun light upon a focus hundreds of meters distant. In this paper a novel sensor design to generate an alignment sensor signal is presented. A detailed study of its response is included, showing that there exist several design parameters to achieve a desired accuracy. This sensor has been implemented using bipolar technology and chip to board bonding, and experimental results obtained in laboratory are given
\end{abstract}

\section{Introduction}

In industry, there exist some applications where an accurate estimation of the position of a light source is needed. That is the case of heliostats in solar concentrating power plants. This device consist of a large mirror that can be rotated respect to a vertical and horizontal axes, named azimuth and elevation axes, as it is shown in Fig. 1. The sunlight is projected upon a focus that is hundreds of meters far from the mirror. Because a maximum projection error of one quarter of meter is permitted, a maximum positioning angle error of one miliradian must be achieved.

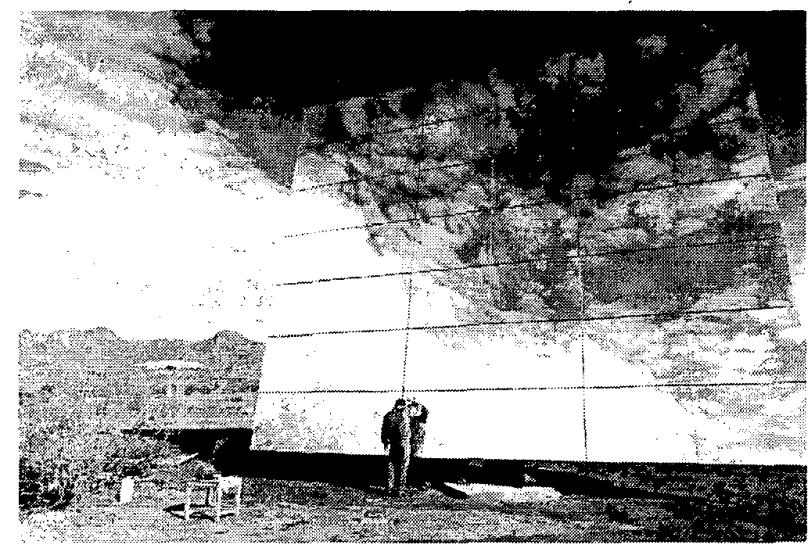

Fig. 1. Photograph of a heliostat.

\author{
M. Domínguez, I. Ameijeiras and L. \\ Castañer \\ Dpto. Ingenieria Electrónica \\ E.S. de Ingenieros de Telecomunicación \\ de Barcelona \\ e-mail: mpumar@eel.upc.es
}

Therefore an accurate control scheme is needed to guarantee a correct positioning of the reflected ray in its aiming point in the receiver.

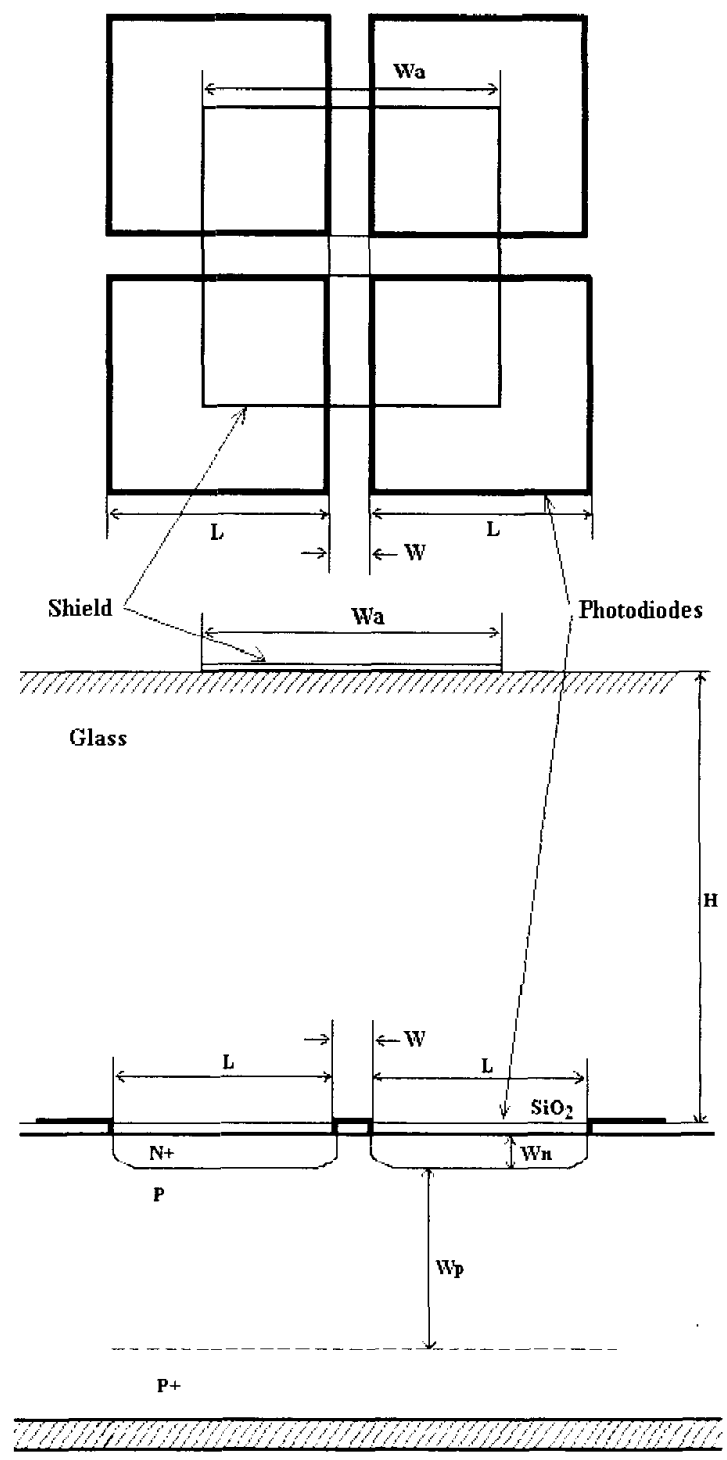

Fig. 2. Structure of the sensor. 


\section{Sensor description}

The sensor consists in four photodiodes disposed in a $2 \times 2$ array partially covered with a metal shield, as presented in Fig. 2. When a light ray falls on the sensor, the shadow of the metal shield is projected over the photodiode array, in such a way that only one fraction of area in each photodiode will be illuminated. The light energy generates a photocurrent that is proportional to the illuminated area of every photodiode. Because this area depends on the shadow projected over the photodiodes, and it also depends on the incident angle of the light ray, we can determine the light source relative position respect to the sensor by measuring the difference of photocurrents that are generated.

The proposed sensor allows the detection of the position of the light source respect to perpendicular planes simultaneously, simply adding currents in pairs.

There exist some alternative schemes [2] that compare the total incident light over two cells. In the proposed sensor, the position of the shadow over the array generates a positioning error signal. As it will be demonstrated later on, the distance $(\mathrm{H})$ between the metal shield and the photodiodes is a parameter that multiplies the sensitivity of the device.

In order to have a close loop feedback control for the heliostat, the use of the proposed sensor to analyse the reflected ray is suggested, at it is shown in Fig. 3 . The sensor is initially adjusted using a laser ray coming from the focus. The sensor is rotated in both axes to achieve zero differential currents in the sensors. Using this procedure we eliminate any geometry deviation generated during the manufacturing and the installation of the sensor.

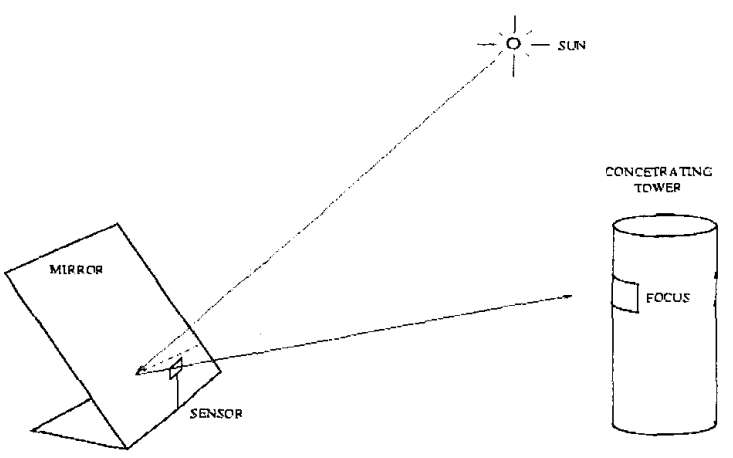

Fig. 3. Application scheme of the sensor controlling a heliostat.
From the sensor point of view, a rotation of the mirror is seen as a displacement of the mirrored sun. The error signal generated by the sensor can be used to rotate the mirror to align the sun respect to the receiver.

\subsection{The Photodiode Current}

The calculus, of the current generated by the photovoltaic effect has been widely studied in literature [3]. The basic parameter that expresses the resulting efficiency of a photodiode is the spectral response $S_{R}$, that it is defined as the number of carriers to the number of incident photon ratio:

$$
S_{R}=\frac{J_{L}(\lambda)}{q N_{F}(\lambda)(1-R)}
$$

where coefficient $\mathbf{R}$ represents the photon reflection on the semiconductor surface, $J_{L}(\lambda)$ is the spectral density current and $\mathrm{N}_{\mathrm{F}}$.

The carrier generation is the contribution of carriers generated in the three semiconductor regions of the photodiode, named $S_{m}, S_{r p}, S_{r r}$, for the $\mathrm{n}, \mathrm{p}$ and depletion region respectively:

$$
S_{R}=S_{m}+S_{, p}+S_{r}
$$

These parameters can be obtained integrating the continuity equation to obtain the minority carrier concentrations in each region.

$\mathrm{J}_{\mathrm{L}}(\lambda)$ can be obtained from equation (1), and the total photocurrent can be calculated by integrating that expression over all the spectral range:

$$
I_{L}=q A \int_{0}^{\lambda_{g}} N_{F}(\lambda)(1-R) S_{R}(\lambda) d \lambda
$$

where $A$ is the area of the light beam and $\lambda \mathrm{g}$ the cutoff wavelength of the semiconductor.

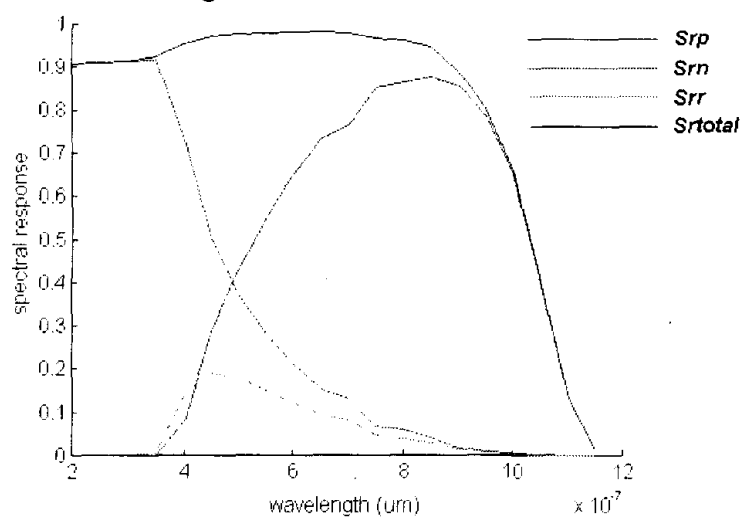

Fig. 4. Spectral response components 


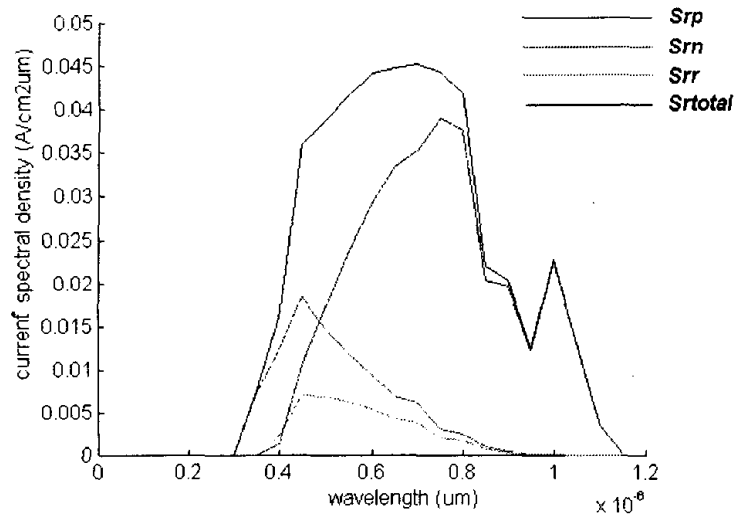

Fig. 5. Current components

The spectral response and current components in the photodiode are depicted in Figs. 5 and 6 respectively, using the following technological parameters: $\mathrm{N}_{\mathrm{d}}=5 \times 10^{18} \mathrm{~cm}^{-3}, \mathrm{~N}_{\mathrm{a}}=10^{16} \mathrm{~cm}^{-3}, \mathrm{Wn}=0.5$ $\mu \mathrm{m}, \mathrm{Wp}=250 \mu \mathrm{m}$.

\subsection{Device Modelling}

The behavior of the device is determined by the projection of the shadow over the array of photodiodes. When the sensor is pointing at the light ray, this projection is perpendicular, at it is illustrated in Fig. 6

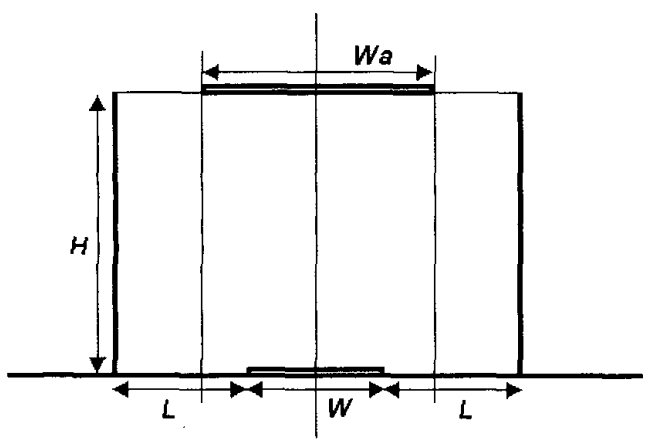

Fig. 6. Device pointing at the light source

The illuminated area in each photodiode is the same. Because the generated photocurrent is proportional to that area, they are in balance, and the differential signal generated by the sensor is null (see Fig. 7). A deviation from this position produces a deviation in the incident angle (see Fig. 8).

In order to obtain an error signal corresponding to the angular deviation in one axis, the photocurrents have to be added in pairs: $(1+3)-(2+4)$ for the vertical axis and $(1+2)-(3+4)$ for horizontal one. In this way, the influence of the deviation in the other axis is cancelled out.

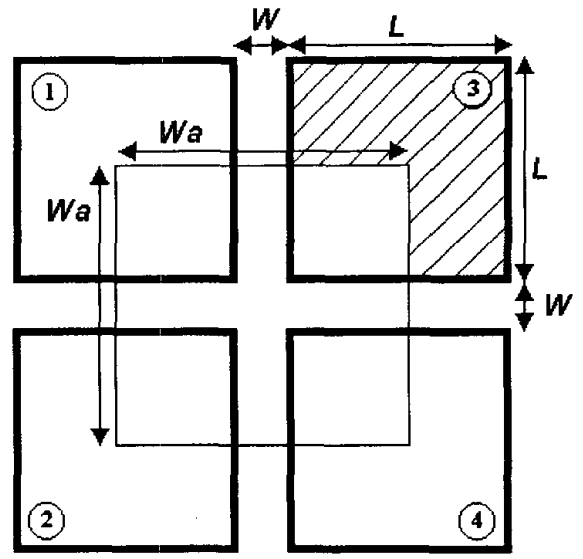

Fig. 7. Front view of the sensor when it is perpendicular to the light source

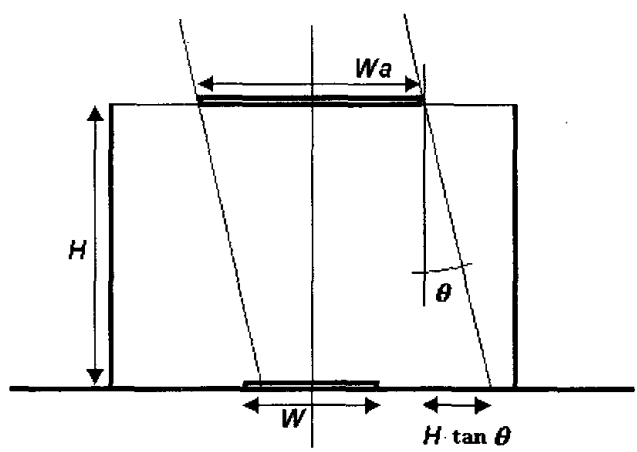

Fig. 8. Front view of the sensor with a deviation in the incident angle
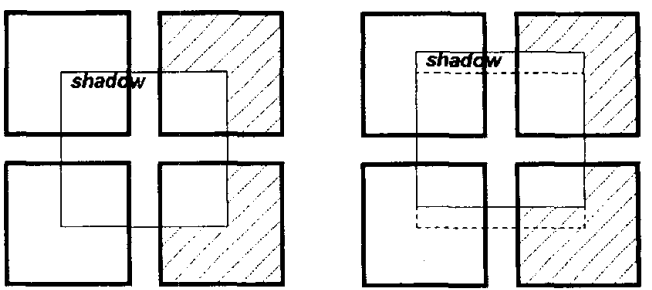

Fig. 9. Displacement of the shadow over the photodiodes

The increment of illuminated area in each pair of photodiodes is given by:

$$
\Delta A=H \cdot \tan \theta \cdot(W a-W)
$$

and the current difference is:

$$
\Delta I=2 \cdot H \cdot \tan \theta \cdot(W a-W) J_{L}
$$

The incident angle $\theta$ can be obtained from equation (3) as a function of the current difference. 


$$
\theta=\arctan \left(\frac{\Delta I}{2 H(W-W a) J_{L}}\right)
$$

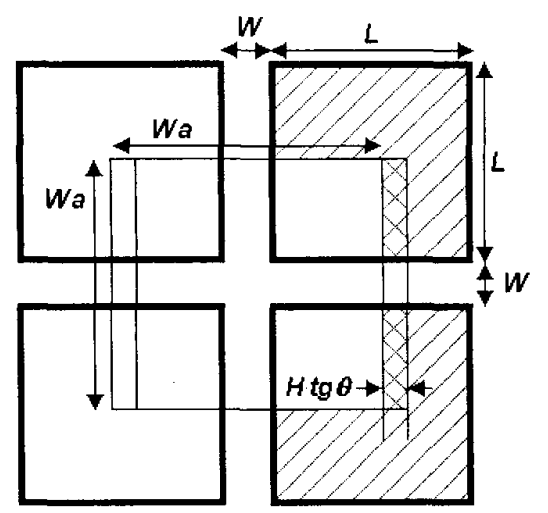

Fig. 10. Detailed displacement of the shadow over the photodiodes

There exists a trade-off between the sensitivity of the sensor and the maximum deviation angle that can be measured. On one hand, an increase in Wa provides a larger range of measure. On the other hand, that increase also represents a lower gain factor, according to eq. 4, and therefore less accuracy.

According to the static response of the sensor previously determined, the following geometrical parameters have been chosen: $H=2000 \mu m, W a=$ $3500 \mu \mathrm{m}, \mathrm{L}=2000 \mu \mathrm{m}, \mathrm{W}=400 \mu \mathrm{m}$. In Fig. 11 , a photograph of the sensor die is shown.

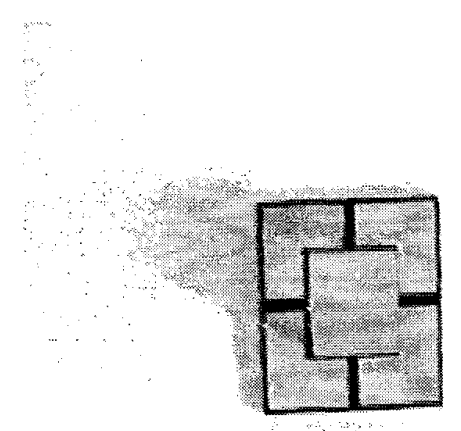

Fig. 11. Photograph of the sensor die

A signal conditioning circuit including current to voltage conversion of the difference of currents has been employed. The sensor has been exposed to direct sun light to evaluate its performance. The experimental response of this sensor is drawn in Fig
12. Notice that for one miliradian the difference in the electrodes is $3 \mathrm{mV}$, providing a sufficient resolution for an accurate angle positioning.

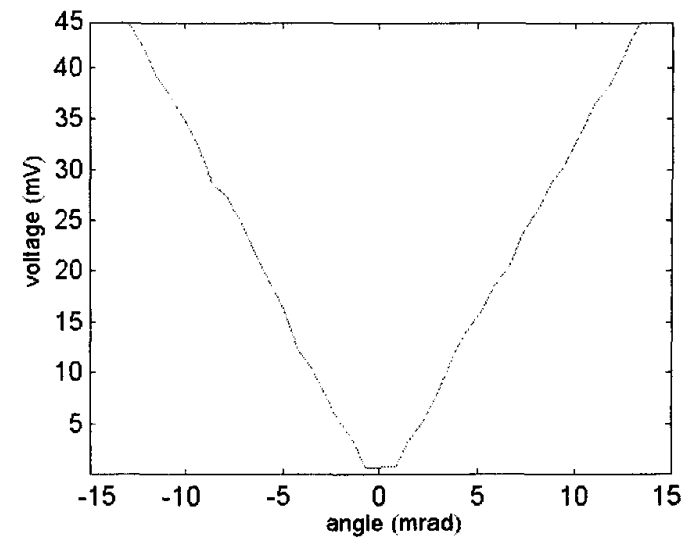

Fig. 12. Voltage output signal vs. Incident angle of the light ray

Two commercial versions of this sensor are currently being studied, one using SENSONOR technology [5] and another with Fagor Electrónica technology. They will include the signal conditioning circuit and ADC converter to provide a digital interface to the control circuit of the heliostat.

\section{Conclusions}

A novel sensor design to accurately determine the position of a light source has been introduced. This structure consists in a photodiode array covered by a metal shield cover that projects a shadow over them. A detailed analysis of the sensor has been developed, and it is remarkable that the distance from the aperture to the sensor plays a crucial role in the design, and it can be regarded as a gain factor in the position sensor. As a result, a desired sensibility can be achieved placing the aperture sufficiently far form the photodiode. Other design parameters guarantee robustness against noise and fabrication tolerances. This design is patent protected [6].

\section{References}

[1] Michalsky J. J. "The astronomical almanac's algorithm for approximate solar position (19502050)". Solar Energr: Vol. 40. №3, pages 227-235. 1988.

[2] Graeme J. Photodiode Amplifiers, McGrav Hill, 1995.

[3] Liou J.J., Advanced Semiconductor Device Physics and Modeling. Artech House. 1994.

[4] Valette S. Renard S. Jadot P., Gidon P., Erbeia C. "Silicon-Based Integrated Optics Technology for Optical Sensor Applications.". Sensor and Actuators. A21-23, pages 1087-1091. 1990

[5] Normic MPW Process Design Hadbook. V 2.0. 1999.

[6] Patent number P9901375 